\title{
Methicillin resistant staphylococci associated with bovine mastitis and their zoonotic importance
}

S. Vishnupriya ${ }^{1}$, P. X. Antony ${ }^{1}$, H. K. Mukhopadhyay ${ }^{1}$, R. M. Pillai ${ }^{1}$, J. Thanislass ${ }^{2}$, V. M. Vivek Srinivas ${ }^{1}$ and R. Sumanth Kumar ${ }^{1}$

1. Department of Microbiology, Rajiv Gandhi College of Veterinary and Animal Sciences, Pondicherry University, Pondicherry - 605 009, India; 2. Department of Veterinary Biochemistry, Rajiv Gandhi College of Veterinary and Animal Sciences, 605 009, India

Corresponding author: V. M. Vivek Srinivas, Present address: FMD Vaccine Research Laboratory, Indian Veterinary Research Institute (IVRI), Hebbal, Bangalore-560 024, India. Ph: +9197428 91992, email: vivekvet24@gmail.com SV: vishnupriyavet@gmail.com, PXA: pxantony@gmail.com, HKM: hkmparvo@gmail.com, RMP: drrmadhu@sify.com, JT: jthanislass@gmail.com, RSK: sumanth.vetco@gmail.com

Received: 13-03-2014, Revised: 03-05-2014, Accepted: 08-05-2014, Re-revised: 24-06-2014, Published online: 25-06-2014

doi: 10.14202/vetworld.2014.422-427 How to cite this article: Vishnupriya S, Antony PX, Mukhopadhyay HK, Pillai RM, Thanislass J, Vivek Srinivas VM and Sumanth Kumar R (2014) Methicillin resistant staphylococci associated with bovine mastitis and their zoonotic importance, Veterinary World 7(6): 422-427.

\begin{abstract}
Aim: The present study was conducted to determine the zoonotic importance of methicillin resistant staphylococci associated with bovine mastitis and their potential role in transmission to animal handlers.

Materials and Methods: A total of 158 milk samples from bovine mastitis cases and 126 nasal swabs from the animal handlers were sampled in and around Pondicherry (Southern India). The Presence of Staphylococcal organism was confirmed by PCR amplification using the genus specific primers and among the isolated Staphylococci; methicillin resistance was identified by genetic amplification of mec $A$ methicillin resistant gene. Then the amplified gene from the bacteria expressing the mec $A$ gene (PBP2a) ( $2 \mathrm{~kb}$ fragment) was further sequenced using four sets of primer pairs and aligned for determining their genetic relatedness between the sequences. Both phenotypic and genotypic analysis was carried out for the six MRS isolates (three bovine and three human) in this study.
\end{abstract}

Results: Out of 158 mastitis milk samples; 96 and 19 bovine isolates were found to be positive for Staphylococcal genus specific PCR and methicillin resistant $(m e c A)$ gene PCR, respectively. Similarly, Out of 126 human nasal swabs, 64 and 13 human isolates were found to be positive for Staphylococcal genus specific PCR and mec $A$ gene PCR, respectively. Among the 160 staphylococcal isolates (Bovine and Human origin); 51 were identified as coagulase-positive staphylococci (CPS) and remaining as coagulase-negative staphylococci (CONS). The results obtained in this study revealed the presence of many species of Staphylococci but the predominant species were Staphylococcus aureus and S. epidermidis. The Sequence analysis of the $m e c A$ gene of human isolates obtained in this study had a maximum identity $(99 \%-100 \%)$ with the bovine isolates.

Conclusion: The phenotypic and genotypic analysis carried out for the six Methicillin Resistant Staphylococci (MRS) isolates in this study were indistinguishable and epidemiologically related, which may indicate the transmission of MRS between bovine and humans. The occurrence of methicillin resistance among staphylococci isolated from cases of bovine mastitis is increasing, necessitating the periodic surveillance for antimicrobial resistance patterns of Staphylococci in order to control the spread of MRS.

Keywords: bovine mastitis, coagulase-negative staphylococci (CONS), methicillin resistant Staphylococcus aureus (MRSA), sequence analysis.

\section{Introduction}

Mastitis is the most common infectious disease among dairy cattle and has been singled out as the most significant cause of economical loss in the dairy industry. Control of mastitis is important because milk may harbor organisms potentially pathogenic to humans [1]. Staphylococci are the bacteria most commonly isolated from subclinical mastitis [2]. However, during recent years, coagulase-negative staphylococci (CONS) have become the most common bovine mastitis isolates in many countries and are regarded as emerging mastitis pathogens [3]. The indiscriminate treatment with antibiotics, without either a technical prescription or identification of the pathogen, can contribute to an

Copyright: The authors. This article is an open access article licensed under the terms of the Creative Commons Attribution License (http://creativecommons.org/licenses/by/2.0) which permits unrestricted use, distribution and reproduction in any medium, provided the work is properly cited. increased resistance of these organisms, making the cure of mastitis still more difficult.

More than $80 \%$ of Staphylococcus aureus strains produce penicillinase, and thus $\beta$-lactam antibiotics such as methicillin, which are resistant to penicillinases, were widely used to treat $S$. aureus infections. By 1990s, Methicillin Resistant Staphylococcus aureus (MRSA) had become a serious nosocomial infection worldwide [4]. MRSA strains are resistant to $\beta$-lactam antibiotics, including all penicillinase-stable $\beta$ lactams, with resistance most commonly mediated by mec $A$ gene. The mec $A$ gene resides on a genomic island termed the Staphylococcal Cassette Chromosome mec (SCCmec) [5]. This gene encodes for a penicillin-binding protein (PBP2a) which is expressed in the bacterial cell wall and has a low affinity for $\beta$ lactam antibiotics. Thus, this group of antibiotics is ineffective against bacteria expressing mec $A$ gene. In 
Table-1: Details of samples/isolates in this study

\begin{tabular}{lccccc}
\hline Sample & $\begin{array}{c}\text { No. of } \\
\text { samples }\end{array}$ & $\begin{array}{c}\text { Gram positive } \\
\text { cultures }\end{array}$ & $\begin{array}{c}\text { Staphylococcus genus specific PCR } \\
\text { positive }\end{array}$ & \multicolumn{2}{c}{\begin{tabular}{c} 
MRSA \\
\cline { 3 - 5 }
\end{tabular}} \\
\cline { 3 - 6 } & & 128 & 22 & 74 & 8 \\
coagulase-positive coagulase-negative & coagulase-positive coagulase-negative \\
\hline Hovine mastitis milk & 158 & 88 & 29 & 35 & 6 \\
\hline
\end{tabular}

Table-2: Oligonucleotide primer sequence used for amplification of the following target gene in this study.

\begin{tabular}{|c|c|c|c|c|}
\hline Primers & Primer sequence & Target gene & Size (bp) & Reference \\
\hline MECA-F & 5'GTAGAAATGACTGAACGTCCGATAA 3' & & & \\
\hline MECA-R & $5^{\prime}$ CCAATTCCACATTGTTTCGGTCTAA 3' & mecAgene & 310 & [14] \\
\hline STAP-F & 5' AACTCTGTTATTAGCGAAGAACA3' & Genus specific & & \\
\hline STAP-R & 5' CCACCTTCCTCCGGTTTGTCACC 3 ' & 16S rRNA & 756 & [12] \\
\hline $\begin{array}{l}\text { S.ARS-F } \\
\text { S.ARS-R }\end{array}$ & 5'GCGATTGATGGTGATACGGT 3' & & & \\
\hline $\begin{array}{l}\text { S.ARS-R } \\
\text { F1 }\end{array}$ & $\begin{array}{l}\text { 5'AGCCAAGCCTTGACGAACTAAAGC 3' } \\
\text { 5'AGTTGTAGTTGTCGGGTTTGG 3' }\end{array}$ & nuc gene & 270 & [13] \\
\hline R1 & 5' GGCCAATTCCACATTGTTTC 3' & mecAgene & 454 & [15] \\
\hline F2 & 5' TCCAGGAATGCAGAAAGACC $3^{\prime}$ & & & \\
\hline $\mathrm{R} 2$ & 5' TCACCTGTTTGAGGGTGGAT 3' & & 675 & \\
\hline F3 & 5' GGCTATCGTGTCACAATCGTT 3' & & & \\
\hline R3 & 5' TCACCTTGTCCGTAACCTGA 3' & & 689 & \\
\hline F4 & 5'TCAGGTTACGGACAAGGTGA3' & & & \\
\hline R4 & 5'CGGAGAAGAAGTTGTAGCAGGA 3' & & 715 & \\
\hline
\end{tabular}

the recent years, MRSA has been increasingly reported as an emerging problem in veterinary medicine. Given the importance of $S$. aureus as a cause of mastitis in cattle and the widespread usage of intramammary antibiotics in lactating animals it is perhaps not incorrect to assume that MRSA could be common in bovines.

Data regarding the prevalence of Staphylococcal mastitis in Pondicherry indicated that approximately $50 \%$ of mastitis in cattle is caused by Staphylococci [6]. Methicillin resistance has been reported in Staphylococci from the cases of bovine mastitis in Pondicherry [7]. So Milk could be a source of MRS infection to human beings. Prevalence of MRS among the two groups namely healthy medical students and healthy nursing students belonging to Pondicherry were found to be $8 / 19(42.1 \%)$ and $2 / 21(9.5 \%)$ respectively [8]. The occurrence of MRS infections in both animals and humans are reported in Pondicherry. However no systematic study is available on the relatedness of MRS between animals and humans and vice-versa.

Hence this study was planned for isolation, characterization and molecular detection of methicillin resistance in Staphylococci from cases of bovine mastitis and persons handling the infected cattle; and also to determine the genetic relationship between the animal and human isolates of MRS, to understand the role of animals as reservoir for MRS infection to humans and vice-versa.

\section{Materials and Methods}

Isolation and identification of Staphylococci: A total of 158 milk samples from mastitis affected animals and 126 nasal swabs from animal handlers (in contact persons) were sampled in Pondicherry (Southern India) (Table-1) aseptically in sterile vials and cultured in Mueller Hinton agar/blood agar for isolation of the organism. The isolated Gram positive bacteria were identified upto genus level as Staphylococci based on the morphology, cultural and biochemical reactions.
All the staphylococcal isolates were identified upto species level based on the methods described by Barrow [9] and William [10].

Antimicrobial sensitivity test: Antimicrobial sensitivity test was done as per the Bauer's standard disc diffusion method [11]. The test was carried out using 16 antimicrobial agents. The interpretation of zone diameter was carried out according to Clinical Laboratory Standard Institute (CLSI). The inoculated plates were inverted and incubated at $37^{\circ} \mathrm{C}$ and each plate was examined after incubation for $48 \mathrm{hrs}$. The diameters of the zones of complete inhibition, including the diameter of the disc, were measured to the nearest whole millimeter with ruler in nonreflecting background. The zone margin was the area where no obvious growth was visible and the readings were recorded.

Genus and species specific PCR: Gram positive cocci which were oxidase negative and catalase positive were subjected to PCR analysis using Staphylococci genus specific primers targeting the 16s rRNA gene of Staphylococci [12] (Table-2). Isolates which were positive by genus specific PCR analysis was subjected to another round of PCR using species specific primers targeting the thermonuclease gene (nuc gene) of $S$. aureus [13]. Detection of methicillin resistance in all the Staphylococcal isolates was carried out by genetic amplification of mec $A$ gene [14] (Table-2).

Genetic amplification of $\boldsymbol{m e c} \boldsymbol{A}$ gene: PCR assay was carried out for amplification of entire mec $A$ gene using four different sets of primer pairs (Figure-1) as per the method described by Malik [15] to determine the genetic relatedness between the MRS isolates of human and animal origin. Then the amplified ( $2 \mathrm{~kb}$ fragment) mec $A$ gene was further sequenced using the same four sets of overlapping primer pairs and aligned to determine their genetic similarities between the sequences. 


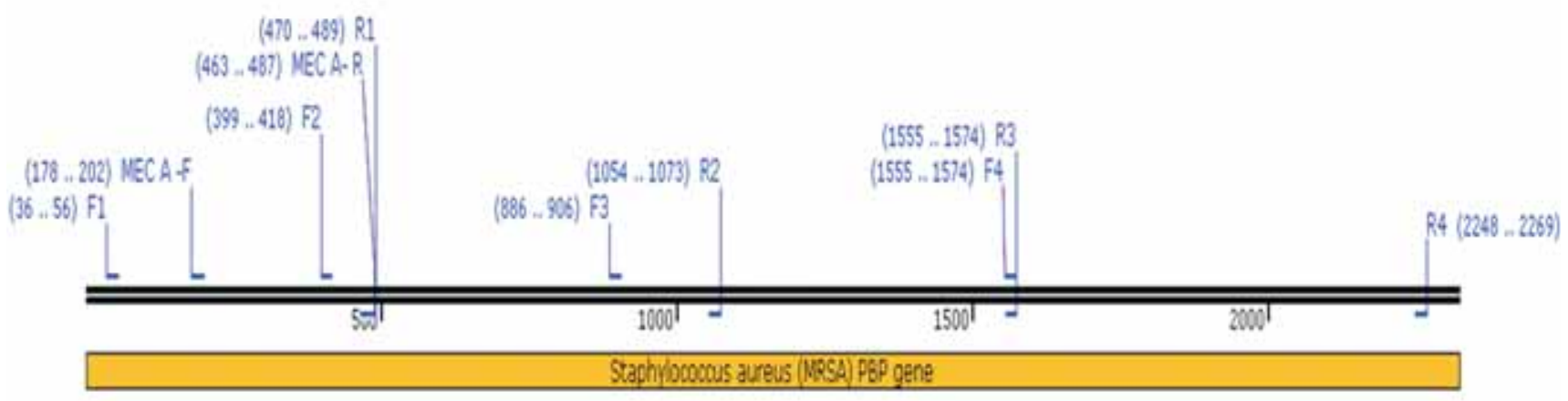

Figure-1: Schematic representation of primer annealing site at mec $A$ gene and the fragment length covered (Developed using SnapGene software).

Sequence and phylogenetic analysis: The four amplified PCR products targeting different regions of the entire mec $A$ gene of six isolates (3 bovine and 3 human isolates) were gel purified using a DNA extraction kit (Eppendorf) and custom sequenced for both directions ( $5^{\prime}-3^{\prime}$ and $\left.3^{\prime}-5^{\prime}\right)$ with same four sets of primer pairs, using the Automated Sequencer, Applied Biosystem 3100. The specificity of the sequences obtained, the nucleotide variations and amino acid variations with respect to the mec A gene sequence of MRSA strain were determined using BLAST [Basic Local Alignment Search Tool] (http://blast.ncbi.nlm. nih.gov/Blast.cgi). The obtained nucleotide sequences of mec A gene were aligned with the sequence of MRSA strain (Y00688) using ClustalW (www.ebi.ac. uk/clustalw) and analyzed for the nucleotide variation of mec Agene.

For phylogenetic analysis, sequences from various parts of the world were retrieved from the GenBank and used. The sequences were aligned using ClustalW 1.8 program and .aln and .nxs files were generated. The .aln file was converted to .meg file using Mega 4 [30] and Neighbor Joining tree (NJ tree) was constructed (bootstrap replicates $=1000$; seed=64,248) using Kimura 2 parameter method for pairwise deletion at uniform rates.

The mec $A$ gene of 6 complete aligned sequences (three human and three bovine isolates) under study were submitted in Genbank under the accession numbers JF710610, JF710611, JF710612, JF710613, JF710614 and Jf710615.

\section{Results and Discussion}

Isolation and identification of Staphylococci from milk samples: Out of the 128 gram positive cultures, 96 were found to carry the Staphylococcus genus specific gene. Among the 96 genus specific PCR positive isolates, 22 were coagulase-positive Staphylococci (Table-1). In this Twenty two, 20 were identified as $S$. aureus and two were $S$. intermedius. These results are in accordance with the study conducted by Amsaveni [6]. Remaining 74 coagulase-negative Staphylococci were characterized upto the species level. They were identified as $S$. epidermidis (17), S. hominis (8), $S$. lugdenensis (6), S. chromogenes (6), S. saccharolyticus (5), S. lentus (5), S. xylosus (4), S. simulans (4), S. hemolyticus (4), S. auricularis (3), S. sciuri (3), S. arlettae (3), S. caprae (2), S. caseolyticus (2), S. capitis (1) and S. cohnii (1).

Isolation and identification of Staphylococci from nasal swabs of animal handlers: Out of the 88 gram positive cultures, 64 were found to carry the Staphylococcus genus specific gene. Among the 64 genus specific PCR positive isolate, 29 were coagulasepositive Staphylococci (Table-1). Among these 29 isolates, 28 were identified as $S$. aureus and one was $S$. intermedius. Remaining 35 coagulase-negative Staphylococci were identified as $S$. epidermidis (12), $S$. lugdenensis (7), S. caseolyticus (3), S. chromogenes (3), S. xylosus (2), S. hominis (2), S. capitis (2), S. auricularis (1), S. caprae (1), S. hemolyticus (1) and $S$. lutrae (1). The present findings are in accord with the study conducted by Ohara who reported that seven species of CONS along with eight $S$. aureus strains were isolated from 18 nasal swabs collected from humans for bacteriological study [16].

Antibiogram of bovine mastitis milk isolates: Out of 22 coagulase-positive Staphylococci, 63.64\% were sensitive to ceftriaxone, $59.09 \%$ were sensitive to chloramphenicol and gentamicin, 50\% were sensitive to enrofloxacin, cloxacillin and ciprofloxacin, and $45.54 \%$ were sensitive to tetracycline, methicillin and oxacillin. $81.82 \%$ were resistant to ampicillin, $72.78 \%$ were resistant to amoxycillin, $63.64 \%$ were resistant to penicillin $\mathrm{G}, 54.54 \%$ were resistant to methicillin and oxacillin, and $50 \%$ were resistant to cloxacillin.

Out of 74 coagulase-negative Staphylococci, $78.38 \%$ were sensitive to chloramphenicol, $75.68 \%$ were sensitive to enrofloxacin, $72.97 \%$ were sensitive to oxacillin and methicillin, $64.86 \%$ were sensitive to co-trimoxazole, $63.51 \%$ were sensitive to gentamicin, $62.16 \%$ were sensitive to ciprofloxacin and cloxacillin. Resistance to ampicillin, amoxycillin, penicillin $\mathrm{G}$, cloxacillin, ciprofloxacin, methicillin and oxacillin were observed in $77.03 \%, 64.86 \%, 52.70 \%, 36.49 \%$, $35.14 \%$ and $27.02 \%$ of the isolates respectively.

Among the 22 coagulase-positive Staphylococci and 74 CONS; 12 CPS and 20 CONS isolates showed methicillin resistance by disc diffusion method (Figure-2). Moon documented that $2.5 \%$ of $S$. aureus and $2.4 \%$ of CONS isolated from bovine mastitis were 


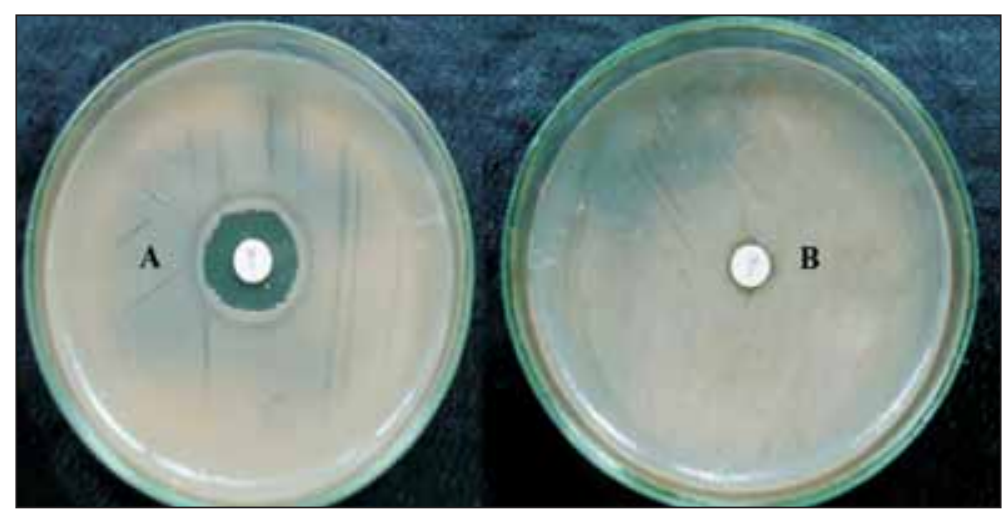

Figure-2: Detection of Methicillin resistance by disc diffusion method. A. Methicillin sensitive staphylococci, B. Methicillin resistant staphylococci.

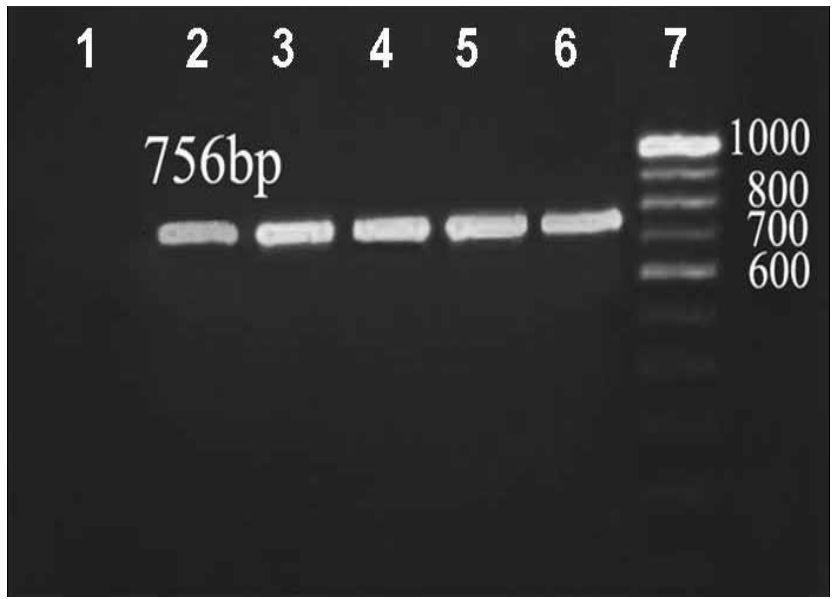

Figure-3: Screening of field isolates for detection of staphylococci using Genus specific $16 \mathrm{~S}$ rRNA binding primer. Lane 1: Negative isolate, Lane 2 to 6: Positive isolates, Lane 7: DNA ladder.

methicillin resistant [17].

Antibiogram of human nasal swab isolates: Out of 29 coagulase-positive Staphylococci, 72.41\% were sensitive to chloramphenicol, $65.51 \%$ were sensitive to cloxacillin, $62.06 \%$ were sensitive to tetracycline, $58.62 \%$ were sensitive to ceftriaxone, and $55.17 \%$ were sensitive to amoxycillin/clavulanic acid, oxacillin and methicillin, $79.31 \%$ were resistant to ampicillin, $55.17 \%$ were resistant to amoxycillin, $48.28 \%$ were resistant to penicillin $\mathrm{G}, 44.83 \%$ were resistant to methicillin and oxacillin and $37.93 \%$ were resistant to cefotaxime.

Out of 35 coagulase-negative Staphylococci, $74.29 \%$ were sensitive to enrofloxacin, $68.57 \%$ were sensitive to cloxacillin and tetracycline, $65.71 \%$ were sensitive to ciprofloxacin, $62.86 \%$ were sensitive to ceftriaxone, and chloramphenicol, $57.14 \%$ sensitive to methicillin and oxacillin. Resistance to ampicillin, penicillin $\mathrm{G}$, amoxycillin (amoxycillin/clavulanic acid, methicillin and oxacillin), cefotaxime were observed in $71.43 \%, 65.71 \%, 62.86 \%, 42.86 \%$ and $34.29 \%$ of the isolates respectively.

Among the 29 coagulase-positive Staphylococci and 35 CONS; 13 CPS and 15 CONS isolates showed methicillin resistance by disc diffusion method (Figure2). Tariq isolated 45 Staphylococci from eighty four

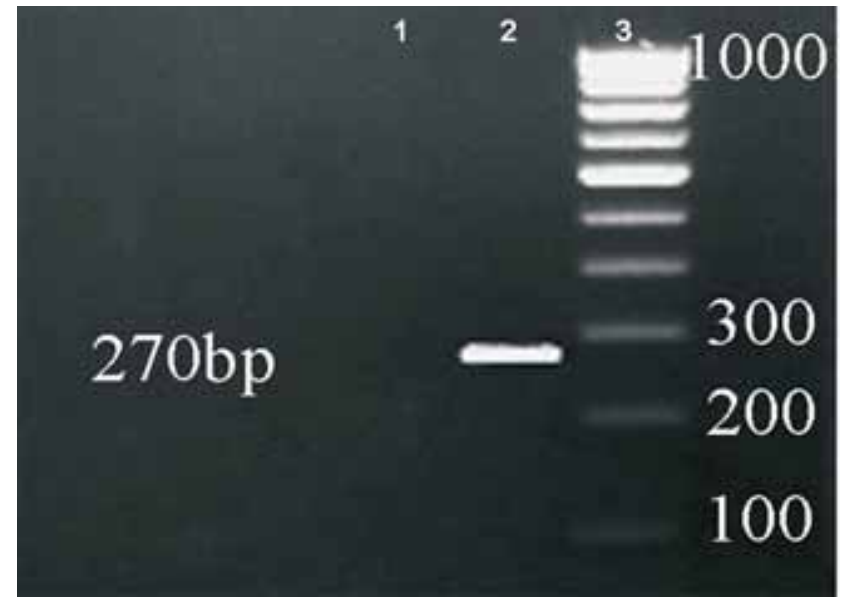

Figure-4: Screening of field isolates for detection of Staphylococci aureus using species specific primers targeting the thermonuclease gene (nuc gene). Lane 1: Negative isolate, Lane 2: Positive isolate, Lane 3: DNA ladder.

swabs taken from the anterior nares of various staff members of the unit. Out of 45 isolates, 35 CONS and 4 $S$. aureus isolates were resistant to methicillin by disc diffusion method [18].

\section{Genetic amplification and sequence analysis}

Genus and species specific PCR: Out of the 128 gram positive isolates from animals suffering from mastitis and 88 gram positive isolates from human nasal swabs subjected to PCR with Staphylococcus genus specific primers, 96 and 64 isolates respectively were confirmed to carry genes specific for Staphylococci targeting a $756 \mathrm{bp}$ fragment within the 16S rRNA gene of Staphylococcus (Figure-3). Among 22 CPS of Bovine mastitis isolates and 28 CPS of Human nasal swab isolates; 20 and 28 isolates respectively gave a single DNA band of approximately $270 \mathrm{bp}$ of the nuc gene confirmed the identity of S. aureus (Figure-4) .

Genetic amplification of mec $\boldsymbol{A}$ gene: Among the 96 bovine mastitis staphylococcal isolates subjected to PCR with mec $A$ gene primers, 8 isolates of $S$. aureus and 11 isolates of CONS (total 19) were detected to be positive for methicillin resistance targeting a $310 \mathrm{bp}$ fragment within the mec $A$ gene of Staphylococcus (Figure-5). Huber demonstrated that out of $142 S$. aureus strains obtained from 2,662 samples collected 


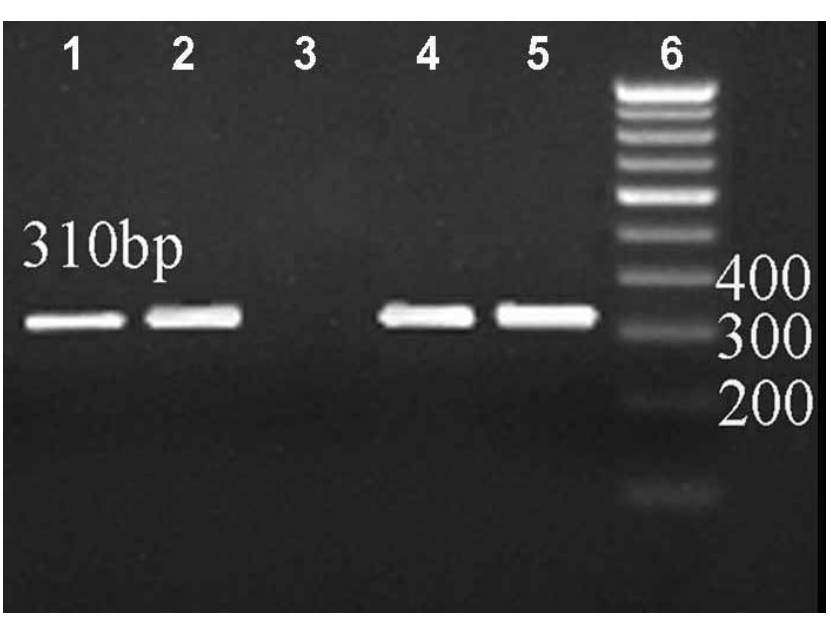

Figure-5: Screening of field isolates for detection of Methicillin resistance of Staphylococci using primers targeting mec A gene. Lane 3: Negative isolate, Lane 1, 2, 4, 5: Positive isolate, Lane 6: DNA ladder.

from bovine mastitis in Switzerland [19], only 2 $(1.42 \%)$ isolates were positive for MRSA. Febler found that out 121 CONS isolated from cases of bovine mastitis, 16 isolates $(13.22 \%)$ were classified as methicillin resistant and 96 isolates as methicillin susceptible by disc diffusion method. But the mec $A$ gene was identified only in 15 out of 16 resistant isolates $(12.40 \%)$ respectively [20].

Similarly among the 64 human nasal swab staphlococcal isolates; 6 isolates of $S$. aureus and 7 isolates of CONS (total 13) were methicillin resistant as they harbored mec $A$ gene. The results of the present finding are in accordance with Unal [21] who found that out of ninety eight staphylococcal isolates obtained from the nasal swabs of healthy volunteers, fifty one isolates were mec $A$ positive. Among 51 methicillin resistant isolates $17(17.74 \%)$ were $S$. aureus strains and $34(34.70 \%)$ were coagulase-negative Staphylococci which included $S$. epidermidis, S. haemolyticus and S. simulans.

Sequence analysis: Four sets of overlapping primers targeting larger segment of mec $A$ gene were used in this study. Template DNA prepared from three human (JF710610, JF710611, JF710612) and bovine methicillin resistant isolates (JF710613, JF710614, JF710615) were subjected to PCR with these four set of primer pairs. Each primer pair yielded an amplified product of $454 \mathrm{bp}, 675 \mathrm{bp}, 689 \mathrm{bp}$ and $715 \mathrm{bp}$ overlapping segments of the mec $A$ gene (Figure-6). The four PCR products for each of the six isolates (three human and three bovine) were gel purified and custom sequenced.

The aligned nucleotide sequence of mec A gene of three methicillin-resistant Staphylococcal isolates of human (JF710610, JF710611, JF710612) and bovine (JF710613, JF710614, JF710615) obtained using each set of primers were subjected to "BLAST" analysis and found to produce a $98-99 \%$ homology with mec A gene (penicillin binding protein gene) of S. aureus and other species of Staphylococcal strains available in the GenBank.

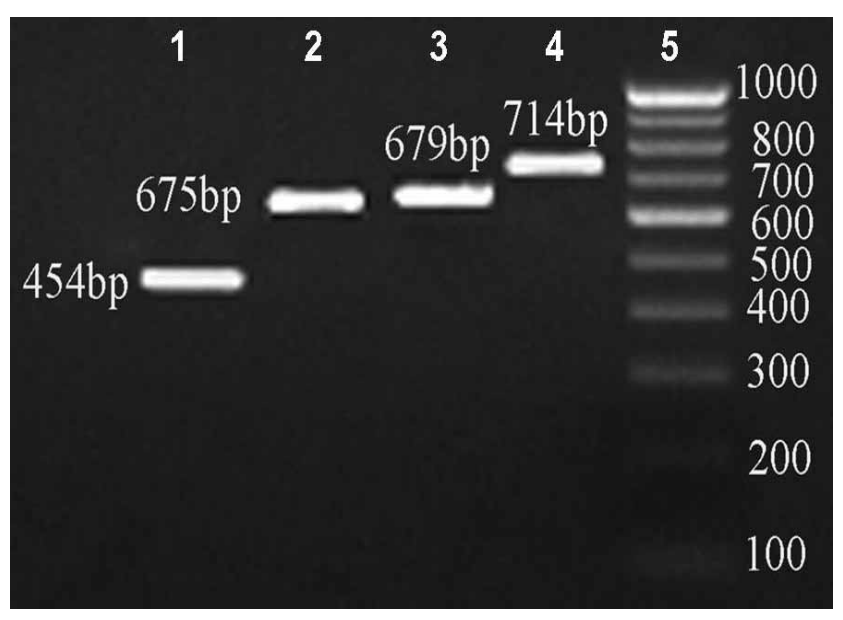

Figure-6: PCR amplification of overlapping segments of mec $A$ gene.

The complete sequence of mec A gene (penicillin binding protein gene) of three human and three bovine isolates were subjected to pair wise blast in order to find the genetic similarity between the isolates of bovine and their related handlers viz I-(JF710610 and JF710613), II-(JF710611 and JF710614) and III(JF710612 and JF710615) were subjected for the pair wise blast. BLAST analysis of mec $A$ gene of human and bovine isolates (JF710610 and JF710613; JF710611 and JF710614) revealed that the sequence had a maximum identity of $100 \%$ while there was $99 \%$ homology between JF710612 and JF710615 isolates. The study is in accordance to the Turutoglu [22] who reported three methicillin resistant $S$. aureus isolates from bovine mastitis cases that were sequenced and found identical to human strains. Similar results showing homology between mec $A$ gene from dogs and cats [15], horses [23] and humans have been reported. Based on the results obtained from this study and other reports [15], it is presumed that these isolates may originate from humans, considering that the methicillin resistance among human $S$. aureus isolates is more common.

Phylogenetic analysis: Phylogenetic analysis of the obtained sequences using MEGA 4.1 neighbor-joining method revealed that the nucleotide sequence of the JF710610 (human isolate) were found to be phylogenetically related to JF710610 (bovine isolate) showing distinct lineage and shared ancestral origin with other isolates obtained in this study and with the MRS strains of various species from different parts of the world.

\section{Conclusion}

Both phenotypic and genotypic analysis showed that the three MRS isolates of bovine and human origin were indistinguishable. It could be concluded that the bovine and human MRS strains described in this study were epidemiologically and genetically related, which may indicates the transmission between bovine and humans. Further studies using DNA sequence based 
methods such as SCC mec typing, pulsed-field gel electrophoresis (PFGE), multilocus sequence typing (MLST), and DNA sequencing of the $\mathrm{X}$ region of the protein A gene (spa typing) of these isolates will improve our understanding of the mobile genetic element carrying the mec $A$ gene and the clonal relationship of these bovine MRS isolates to human. This will aid in the understanding the transmission of MRS between bovine and their handlers and will also help in formulating strategies for the control of spread of MRS infections. Periodic surveillance for antimicrobial resistance patterns of MRS isolated from dairy cows with mastitis could be an important measure in detecting the emergence and spreading of such resistance.

\section{Authors' contributions}

SV and PXA were involved in the design of this research work. The research was done by SV. PXA has monitored all the activities being a supervisor. VMVS, RSK, HKM, RMP and JT have assisted this research work. VMVS and SV drafted and revised the manuscript. All authors read and approved the final manuscript.

\section{Acknowledgements}

The authors are grateful to the Dean, Rajiv Gandhi College of Veterinary and Animal sciences, Kurumbapet, Pondicherry, India for providing necessary facilities and fund to conduct this research.

\section{Competing interests}

The authors declare that they have no competing interests.

\section{References}

1. Koch, R., Schaumburg, F., Mellmann, A., Köksal, M., Jurke, A., Becker, K. and Friedrich, A. W. (2013) LivestockAssociated Methicillin-Resistant Staphylococcus aureus (MRSA) as Causes of Human Infection and Colonization in Germany, PLoS ONE, 8(2): e55040.

2. Benhamed, N. and Kihal, M. (2013) Genotypic Characterization of Staphylococcus aureus Involved in Bovine Mastitis in Algeria, Res J Appl Sci, 8(4): 262-267.

3. El-Jakee, J. K., Aref, N. E., Gomaa, A., El-Hariri, M. D., Galal, H. M., Omar, S. A. and Samir, A. (2013) Emerging of coagulase negative staphylococci as a cause of mastitis in dairy animals: An environmental hazard, Int. J. Vet. Sci. Med.,1:74-78.

4. Anon (2001) European Antimicrobial Resistance Surveillance System (EARSS). Annual Report. National Institute of Public Health and the Environment, Bilthoven.

5. Ito, T., Katayama, Y., Asada, K., Mori, N., Tsutsumimoto, K., Tiensasitorn, C. and Hiramatsu, K. (2001) Structural comparison of three types of staphylococcal cassette chromosome mec integrated in the chromosome in methicillin-resistant Staphylococcus aureus, Antimicrob. Agents Chemother, 45: 1323-1336.

6. Amasaveni, A., Antony, P. X., Mukhopadhyay, H. K., Pillai, R. M., Vijayalakshmi, P., Thanislass, J. and Subbareddy, K. V. (2010) Detection of beta Lactamase activity in Staphylococci isolated from bovine mastitis, Indian Vet J, 87(7): 647.

7. Krithiga, N., Amsaveni, A., Antony, P. X., Mukhopadhyay, H. K., Pillai, R. M., Vijayalakshmi, P., Thanislass, J. and Subbareddy, K. V. (2014) Detection of Beta Lactamase Activity in Staphylococci Isolated from Bovine Mastitis, Indian Vet J, 88(3): 81.
8. Shanmugam, (2008) The prevalence, antibiogram and characterisation of staphylococcus aureus including MRSA among the healthy staff, medical students and patients from Sri Manakula Vinayagar Medical College and Hospital (SMVMCH), Puducherry. Project submitted to DSTE.

9. Barrow, G.I. and Feltham, R.K. (1993). Cowan and Steel's Manual for the identification of Medical bacteria.3rd edn. Cambridge University Press. p50-142.

10. Vos, P., Garrity, G., Jones, D., Krieg, N.R., Ludwig, W., Rainey, F.A., Schleifer, K.H., Whitman, W.B. (2009) Bergey's Manual of Systematic Bacteriology: The Firmicutes 2nd edition. NewYork: Springer. 3:1450-51.

11. Bauer, A.W., Kirby, W.M., Sherris, J.C. and Turck, M. (1966) Antibiotic susceptibility testing by standardized single disk method, Am. J. Clin. Pathol, 45: 493-497.

12. Zhang, K., Sparling, J., Chow, B.L., Elsayed, S., Hussain, Z., Chruch, D.L., Gregson, D.B., Louie, T. and Conly, J.M. (2004) New Quadriplex PCR assay for detection of methicillin and mupirocin resistance and simultaneous discrimination of S.aureus from coagulase-negative Staphylococci, J. Clin. Microbiol, 42: 4947-4955.

13. Brakstad, O.G., Aasbakk, K. and Maeland, J.A. (1992) Detection of Staphylococcus aureus by polymerase chain reaction amplification of the nuc gene, J. Clin. Microbiol, 30: 1654-1660.

14. Ryffel, C., Tesch, W., Birch-Machin, I., Reynolds, P.E., Barberis-Maino, L., Kayser, F. H. and Berger-Bachi, B. (1990) Sequence comparison of mec $A$ genes isolated from methicillin-resistant Staphylococcus aureus and Staphylococcus epidermidis, Gene, 94: 137-138.

15. Malik, S., Peng, H. and Barton, M. (2006) Partial nucleotide sequencing of the mec A genes of Staphylococcus aureus isolates from dogs and cats, J. Clin. Microbiol, 44: 413-416.

16. Ohara-Nemoto, Y., Haraga, H., Kimura, S. and Nemoto, T.K. (2008) Occurrence of Staphylococci in the oral cavities of healthy adults and nasal oral trafficking of the bacteria, $J$. Med. Microbiol, 57(1): 95-99.

17. Moon, J.S., Lee, A.R., Kang, H.M., Lee, E.S., Kim, M.N., Paik, Y.H., Park, Y.H., Joo, Y.S. and Koo, H.C. (2007) Phenotypic and genetic antibiogram of methicillin-resistant Staphylococci isolated from bovine mastitis in Korea, $J$. Dairy Sci, 90: 1176-1185.

18. Tariq, B., Fahim, Q., Ikram, A., Naqvi, S.H., Khan, S. and Wiqar, M.A. (2009) Rapid detection of methicillin resistant Staphylococci from the nasal screening swabs by mannitol salt agar with cefoxitin and oxacillin disks, Pak. Armed Forces Med. J, 59 (4): 414-419.

19. Huber, H., Koller, S., Giezendanner, N., Stephan, R and Zweifel, C. (2010) Prevalence and characteristics of meticillin-resistant Staphylococcus aureus in humans in contact with farm animals, in livestock, and in food of animal origin, Switzerland, Eurosurveillance, 15:3.

20. Febler, A.T., Billerbeck, C., Kadlec, K. and Schwarz, S. (2010) Identification and characterization of methicillinresistant coagulase-negative Staphylococci from bovine mastitis, Antimicrob. Chemother, 65: 1576-1582.

21. Unal, S., Hoskins, J., Flokowitsch, J.E., Wu, C.Y.E., Preston, D.A. and Skatrud, P.L. (1992) Detection of methicillinresistant Staphylococci by using the polymerase chain reaction, J. Clin. Microbiol, 30: 1685-1691.

22. Turutoglu, H., Hasoksuz, M., Ozturk, D., Yildirim, M. and Sagnak, S. (2009) Methicillin and aminoglycoside resistance in Staphylococcus aureus isolates from bovine mastitis and sequence analysis of their mec $A$ genes, Vet. Res. Commun, 33: 945-956.

23. Schnellmann, C., Gerber, V., Rossano, A., Jaquier, V., Panchaud, Y., Doherr, M.G., Thomann, A., Straub, R. and Perreten, V. (2006) Presence of new mec $A$ and $m p h(C)$ variants conferring antibiotic resistance in Staphylococcus spp. isolated from the skin of horses before and after clinic admission, J. Clin. Microbiol, 44: 4444-54.

$* * * * * * * *$ 